\title{
Preparation and Characterization of Ibuprofen Nanoparticles by using Solvent/ Antisolvent Precipitation
}

\author{
Mansour Mansouri*,1, Hamid Reza Pouretedal ${ }^{2}$, Vida Vosoughi $^{3}$ \\ ${ }^{I}$ Farabi Pharmaceutical Co., Isfahan, I. R. Iran \\ ${ }^{2}$ Department of Chemistry, Malek-ashtar University of Technology, Shahin-shahr, I. R. Iran \\ ${ }^{3}$ Department of Chemistry, Islamic Azad University, Shahreza branch, Shahreza I. R. Iran
}

\begin{abstract}
Ibuprofen as a non-steroidal anti-inflammatory drug (NSAID) show a low and variable oral bioavailability in water solution due to low aqueous solubility and slow dissolution rate. Reducing ibuprofen particles size is an effective and widely used approach to speed up dissolution by enlarging the effective surface area. The ibuprofen nanoparticles are prepared by solvent/antisolvent method. The prepared nanodrug show the accelerate dissolution rate in water solvent. In comparison with raw drug, the chemical structure of nanosized ibuprofen show not change but the crystallinity is reduced to $300-400 \mathrm{~nm}$. The decreasing of particles size ibuprofen is also due to an increase 2.33 times of dissolution in purified water in first $30 \mathrm{~min}$
\end{abstract}

Keywords: Antisolvent, bioavailability, ibuprofen, nanodrug.

\section{INTRODUCTION}

Ibuprofen is a widely prescribed non-steroidal agent with anti-inflammatory, analgesic and antipyretic properties used to treat rheumatoid arthritis, osteoarthritis and mild to moderate pain. It is also used to reduce fever and relieve headaches, muscle aches, backache and aches from cold [1]. In the pharmaceuticals industry, a large numbers of drugs are insoluble or poorly soluble in water and drugs sparingly soluble in water are quite often highly soluble in organic solvents [2]. The bioavailability (the percentage of the drug absorbed compared to its initial dosage) is limited by this insolubility [3-6]. The drug must first be dissolved in order to be absorbed. Dissolution rate is a function of the surface area of the particles and solubility [7]. The surface area can be determined through the control of the particle size. Therefore, the bioavailability of the water insoluble drugs can be improved by reduction in their particle size (increase in surface area) [8]. In the pharmaceutical industry, also several conventional techniques have been utilized for particle size reduction such as crushing, grinding, milling, spray drying, freeze-drying. The disadvantages of using these conventional techniques are thermal and chemical degradation of products due to high temperatures, high energy requirements, much amounts of consumption solvent, solvent-disposal problems, solvent residues, and broad particle size distributions (PSDs) [9-11]. Rasenack et al. succeeded by mixing alcoholic solution of drugs with polymeric materials to control association and micronize the drugs down to a fraction of micrometer [12]. Ibuprofen shows poor dissolution behavior because of its hydrophobic nature [13]. However, a rapid drug release is preferable,

*Address correspondence to this author at the Farabi Pharmaceutical Co., Isfahan, I. R. Iran; Tel: (+98 913) 126 7185; Fax: (+98 311) 6540272;

E-mail: mansoury.mansour@gmail.com especially for analgesic drug. Reducing drug particles size is an effective and widely used approach to speed up dissolution by enlarging the effective surface area [14]. According to Noyes-Whitney equation, the dissolution rate is proportional to the surface area exposed to the dissolution medium [15]. So, the dissolution rate and the oral bioavailability of ibuprofen from these formulations differed widely, methods were time consuming and costly, and some formulations were bulky with poor flow characteristics and handling difficulties [16-18].

\section{MATERIALS AND METHODS}

\subsection{Materials}

All chemicals used were analytical grade with the highest purity available without any purification. Ibuprofen [2-(4isobutylphenyl)-propionic acid] which is a Non-steroidal anti-inflammatory drug (NSAID), was used as the solute. Ibuprofen is poorly soluble in water and readily soluble in most organic solvents were purchased from Dasan Medichem- South Korea. Sodium dodecyl sulfate (SDS or $\mathrm{NaDS})\left(\mathrm{C}_{12} \mathrm{H}_{25} \mathrm{SO}_{4} \mathrm{Na}\right)$, polyvinyl pyrolidone (PVP), sodium lauryl sulfate (SLS), tween 80 (Polysorbate 80), triethanolamine (TEA) were used as surfactant. Isopropyl alcohol (IPA), acetonitrile, methanol, ortho-phosphoric acid and other solvents were from Merck as grade of HPLC. Other chemicals are analytical grade. Purified water was prepared from water complying with the U.S environmental Protection Agency. The cationic and anionic acid resins were used to purified water and after several times, the conductivity fall down from $1050(\mu \mathrm{s} / \mathrm{cm})$ to $10-15$ $(\mu \mathrm{s} / \mathrm{cm})$. After that the media pass trough reverse osmosis (RO) membrane. The result was a purified water with at most $4.2(\mu \mathrm{s} / \mathrm{cm})$ conductivity and its total organic carbon must be meets the requirements. At this work, we used purified water with conductivity $<1.0 \mu \mathrm{s} / \mathrm{cm}$. 


\subsection{Preparation of Ibuprofen Nanoparticles}

Ibuprofen nanoparticles were prepared by using solvent/antisolvent precipitation technique as an effective technology in preparation of nanodrugs. A certain amount of raw drug of ibuprofen was completely dissolved in water miscible solvent. At this work, the isopropyl alcohol is used as solvent.

According to USP, isopropyl alcohol is categorized in Class 3. Class 3 includes no solvent known as a human hazard at levels normally accepted in pharmaceuticals. However there are no long-term toxicity or carcinogenicity studies for many of the residual solvents in Class 3 .

The obtained drug solution was then injected into the water containing the surfactant of SDS and/or TEA as stabilizer under stirring at $3000 \mathrm{rpm}$. Precipitation of solid drug particles occurred immediately upon mixing. The suspension was centrifuged at $15,300 \mathrm{rpm}$ for $20 \mathrm{~min}$ and washed twice with purified water. The precipitated nanoparticles were oven-dried at $35{ }^{\circ} \mathrm{C}$ for $24 \mathrm{~h}$. The influence of other stabilizers such as polyvinyl pyrolidone (PVP), sodium lauryl sulfate (SLS) and tween 80 was also studied in the proposed procedure.

\subsection{Nanoparticles Characterization}

\subsubsection{Particle Size Distribution (PSD)}

The size of drug nanoparticles was measured immediately after precipitation by dynamic laser light scattering (Nanoparticle size analyzer, Malvern). Before analysis, the drug suspension was diluted by purified water to $0.2 \mathrm{mg} / \mathrm{ml}$.

\subsubsection{Infrared Spectroscopy (IR)}

Shimadzu IR-470 spectrometer was used to record the IR spectrum of the ibuprofen nanoparticles from 400 to 4000 $\mathrm{cm}^{-1}$. The sample was grounded with $\mathrm{KBr}$ and pressed to a suitable size disk for measurement.

\subsubsection{Differential Scanning Calorimetry (DSC)}

Differential scanning calorimetry (DSC) was conducted on DSC 131 SETARAM. The samples were equilibrated at $20{ }^{\circ} \mathrm{C}$ for $30 \mathrm{~min}$. Indium standard was used to calibrate the DSC temperature and enthalpy scale. The powder samples were hermetically sealed in aluminum pans and heated at a constant rate of $3{ }^{\circ} \mathrm{C} / \mathrm{min}$, over a temperature range of 20 $170{ }^{\circ} \mathrm{C}$. Inert atmosphere was maintained by purging nitrogen at the flow rate of $15.8 \mathrm{ml} / \mathrm{min}$, linear velocity 35 $\mathrm{cm} / \mathrm{sec}$ and pressure $24.7 \mathrm{kPa}$.

\subsubsection{Scanning Electron Microscopy (SEM)}

The surface morphologies of the ibuprofen nanoparticles were observed by scanning electron microscopy (SEM - AIS -2100 ) with an accelerating voltage of approximate 1.5-20 $\mathrm{kV}$. Gold coating was performed to prevent charge-up of sample.

\subsubsection{Nano-Ibuprofen Assay}

$50 \mathrm{ml}$ of methanol with concentration of $450 \mathrm{mg}$ of nanoibuprofen at presence of $0.4 \mathrm{ml}$ phenolphthalein solution was titrated with $0.1 \mathrm{M}$ sodium hydroxide until a red color obtained. Then carry out a blank titration. Each $\mathrm{ml}$ of $0.1 \mathrm{M}$ sodium hydroxide is equivalent to $20.63 \mathrm{mg}$ of $\mathrm{C}_{13} \mathrm{H}_{18} \mathrm{O}_{2}$ [19].

\subsubsection{Dissolution Test}

The dissolution profiles of ibuprofen were investigated with a dissolution apparatus (Pharmatest dissolution tester), according to a type II paddle method (USP 32), with the rotation speed of paddle was set on $100 \mathrm{rpm}$ and the bath temperature was kept at $37.0 \pm 0.5{ }^{\circ} \mathrm{C} .100$ milligrams of drug powder was put into the vessel containing $900 \mathrm{ml}$ purified water [20]. To avoid float the powder in the water, we retest dissolution test by apparatus type I, basket for raw and nano-ibuprofen, respectively.

At specific intervals, $0.5 \mathrm{ml}$ aliquot of the dissolution medium was sampled (the pooled sample was replaced with a fresh purified water), filtered (pore size: $0.45 \mu \mathrm{m}$ ) and directly injected to the HPLC system and determine the amount of $\mathrm{C}_{13} \mathrm{H}_{18} \mathrm{O}_{2}$ dissolved from UV absorbance of the sample solution, in comparison with a standard solution having a known concentration of ibuprofen raw drug at 221 $\mathrm{nm}$.

\section{RESULTS AND DISCUSSION}

\subsection{Preparation of Ibuprofen Nanoparticles}

Ibuprofen nanoparticles were prepared by antisolvent precipitation method. For this technique, introduction of the drug solution to the antisolvent generates high supersaturation. This results in fast nucleation rate and produces a large number of nuclei, which reduces the solute mass for subsequent growth. Submicron nanoparticles can thus be obtained provided that the growth of nucleating crystals can be arrested by the stabilizer (surfactant or polymer) through steric or electrostatic mechanism [21-23]. For hydrophobic drugs like ibuprofen, water is most commonly used as the antisolvent. In terms of the solvent, it is beneficial if it can solubilize the drug in large amount and possesses a fast diffusion rate to the antisolvent water; while the stabilizer needs to have good affinity for drug particles and possess a fast diffusion rate and effective adsorption onto the drug particle surface in the water-solvent mixture [24-28]. The effect of SDS and TEA is studied on precipitated ibuprofen particle size. The supersaturated drug was injected to separate solutions of SDS and TEA in water with concentrations of $0.1,0.2,0.3,0.4,0.5,0.6,0.7,0.8,0.9$ and $1.0 \% \mathrm{w} / \mathrm{v}$. The influence of stabilizers amount of SDS and TEA and other stabilizers such as polyvinyl pyrolidone (PVP), sodium lauryl sulfate (SLS), tween 80 , on the precipitated ibuprofen particles size are shown in Fig. (1).

Sodium dodecyl sulfate (SDS) is a general surfactant and its effect on precipitated ibuprofen particles is as steric effect and the latest particle size was obtained approximately 700 $\mathrm{nm}$. While, by using the TEA as an emulsifier and a surfactant with electrostatic activity, the particle size was approximately $600 \mathrm{~nm}$. The effect of simultaneous using of SDS and TEA in various ratios on the particles size is shown in Fig. (2). The best results were obtained on (1:1) ratio of SDS and TEA, due to both staric and electrostatic activity of them. Therefore, SDS/TEA surfactant pair is a crucial way to obtain ibuprofen nanoparticles in 300- $400 \mathrm{~nm}$. 


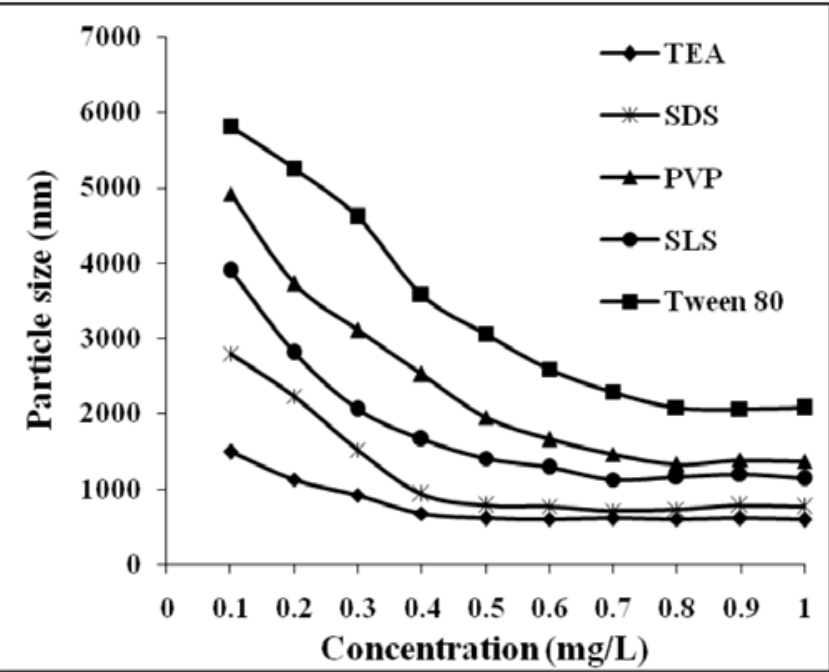

Fig. (1). Influence of kind and concentration of stabilizers on the precipitated ibuprofen particles size.

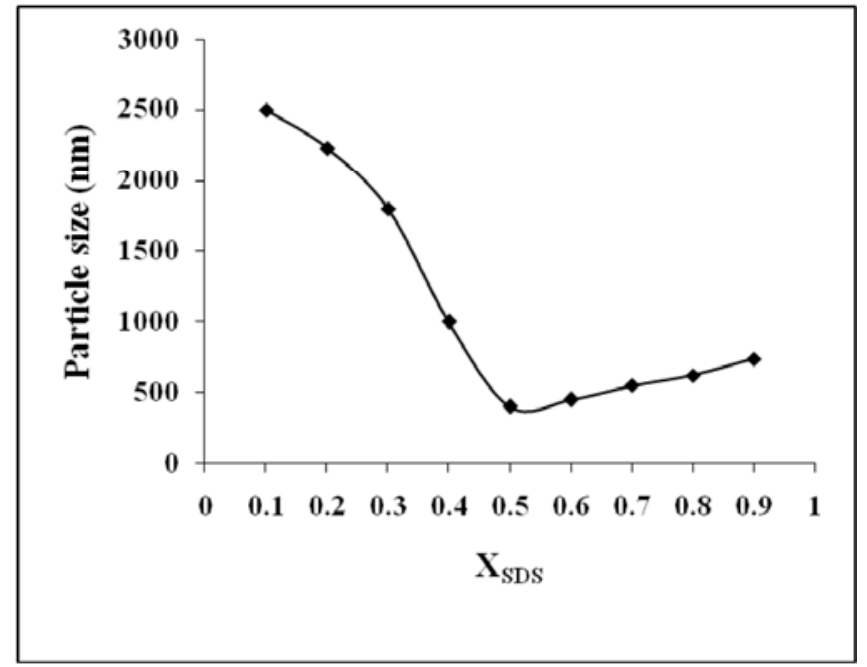

Fig. (2). The effect of volumes fraction of SDS and TEA with the same concentration of $0.5 \% \mathrm{w} / \mathrm{v}$ on the precipitated ibuprofen particles size.

\subsection{Particle Size Distribution}

The particle size of raw ibuprofen is determined between 90- 425 micron (Fig. 3), while the prepared nano-ibuprofen by solvent/antisolvent method shows a narrow particle size distribution at range 200- $450 \mathrm{~nm}$ (Fig. 4). The particles size of raw and nano-ibuprofen was measured by a nanoparticle size analyzer.

\subsection{IR Spectra}

Raw ibuprofen and precipitated nanoparticles exhibit same IR spectrum as shown in Fig. (5), which demonstrates that the chemical structure of the drug is not changed before and after the decreasing of particles size.

\subsection{DSC Analysis}

The physical state of raw ibuprofen and drug nanoparticles was examined by DSC and their thermograms are shown in Fig. (6). Raw ibuprofen exhibits a melting point at $76.20{ }^{\circ} \mathrm{C}$, with fusion enthalpy of $127.72 \mathrm{~J} / \mathrm{g}$. After being

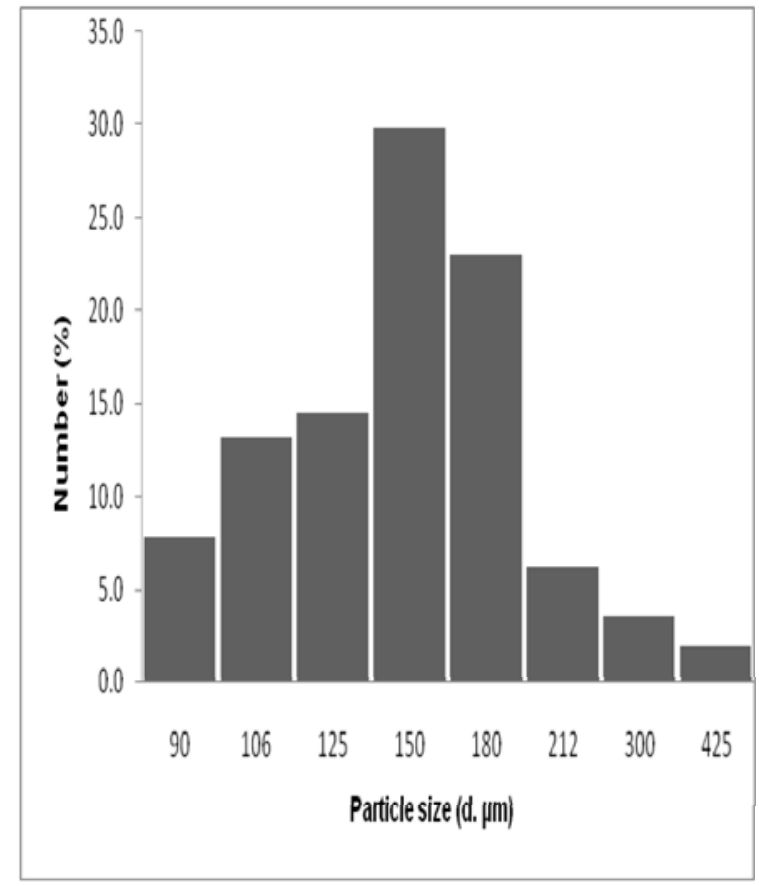

Fig. (3). The distribution of raw ibuprofen particles size.

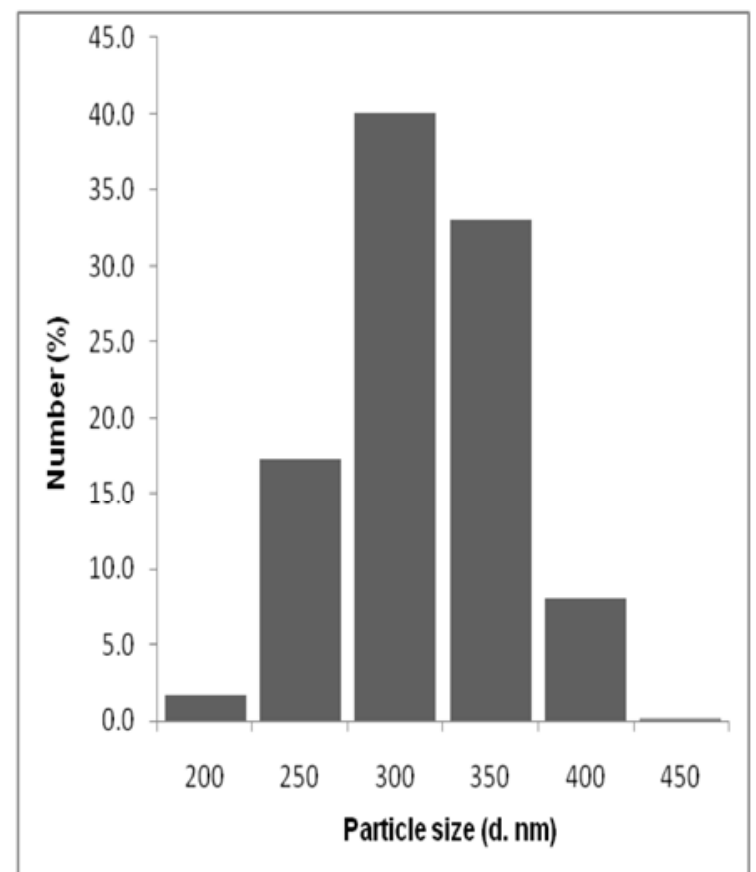

Fig. (4). Particle size distribution of nano-ibuprofen.

precipitated as nanoparticles, its melting point and fusion enthalpy was decreased to $50.42{ }^{\circ} \mathrm{C}$ and $88.98 \mathrm{~J} / \mathrm{g}$, respectively, indicating reduced crystallinity.

\subsection{SEM Scan}

The SEM image and the particle size distribution of the ibuprofen nanoparticles that prepared by solvent/ antisolvent precipitation method, which was obtained from freshly formed nanosuspension, are shown in Figs. (7-a, and 7-b). The morphology of ibuprofen nanoparticles was observed by scanning electron microscopy. SEM images show the size of the particles is in approximate $300-400 \mathrm{~nm}$. 


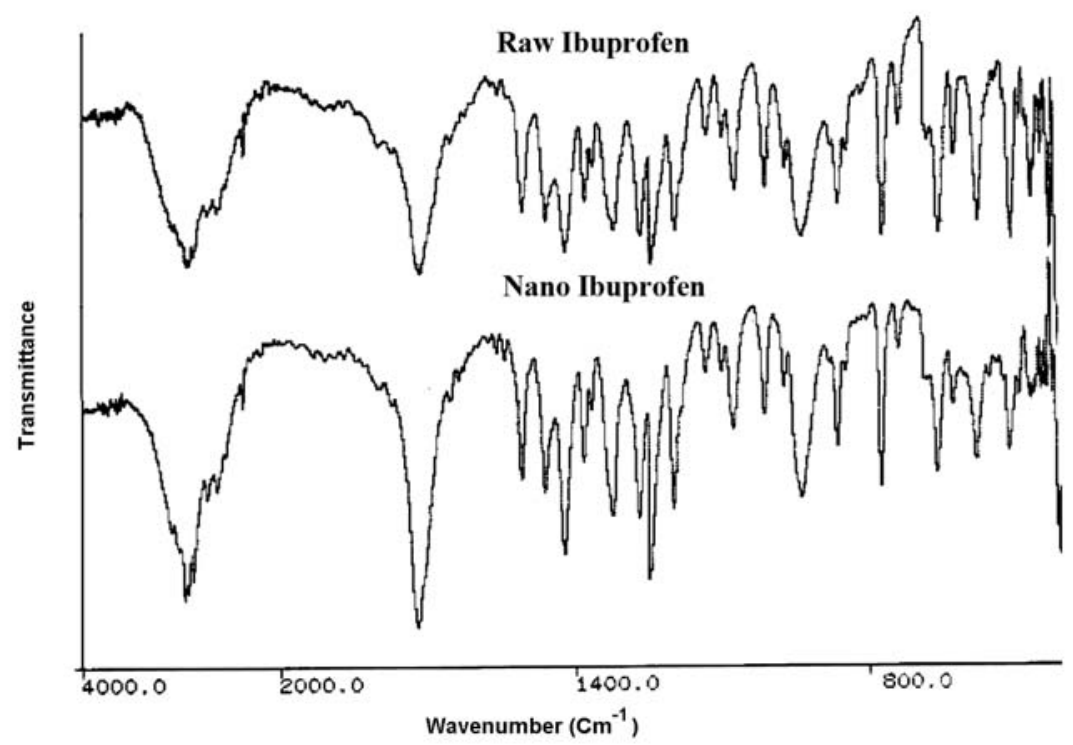

Fig. (5). IR of raw drug and precipitated nano-drug particles.

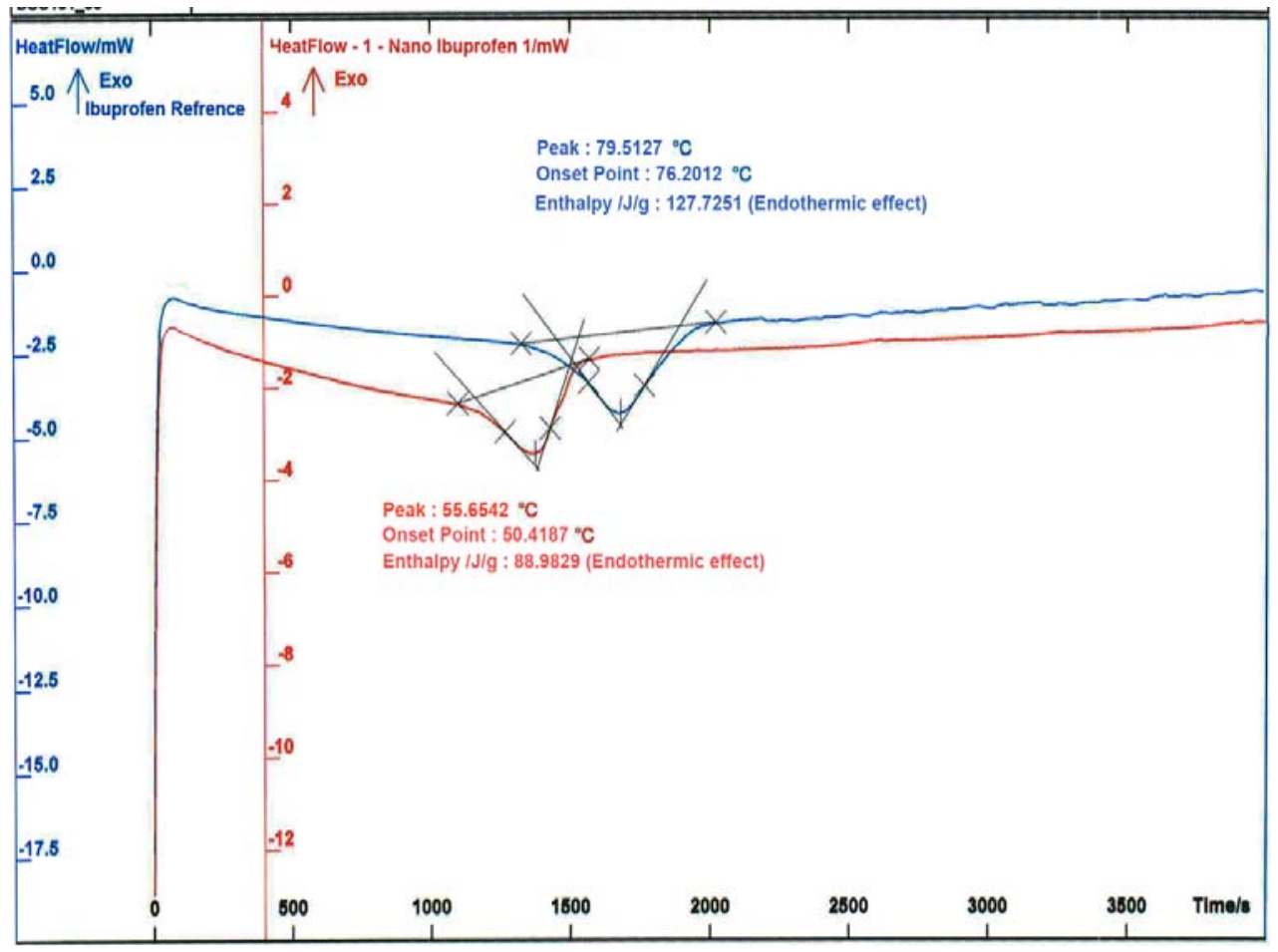

Fig. (6). DSC thermograms of raw ibuprofen and drug nanoparticles.

\subsection{Assay and Chromatographic Impurities}

The purity of precipitated nanoparticles calculated as BP 2010 and show that the precipitated nanoparticles are in highest purity of $99.2 \pm 0.5 \%$. All the results from this calculation are sited in Table $\mathbf{1}$. The impurities of nanoibuprofen are also studied by chromatography method. The mobile phase is prepared by water that adjusted with phosphoric acid to a $\mathrm{pH}$ of 2.5 and acetonitrile (680:1340). The sample solution is prepared with dissolution of $5 \mathrm{mg}$ per $\mathrm{ml}$ of nano-ibuprofen in acetonitrile. The obtained HPLC spectrum is shown in Fig. (8). Individual impurities are not seen more than $0.3 \%$ and sum of all individual impurities is not exceed $1.0 \%$. After that the percentage of each impurity calculated by formula of $100\left(r_{i} / r_{t}\right)$, in which $r_{i}$ is the response of an individual peak, other than the main ibuprofen peak, and $r_{t}$ is the sum of the responses of all the peaks. All the data from this analysis are sited on Table 2. The GC chromatogram of nano-ibuprofen is not show of any impurity such as isopropyl alcohol and hence the nano-drug is completely separated from isopropyl alcohol solvent. Also the specific optical rotation of precipitated nano-ibuprofen shows that the optical rotation of precipitated nano-drug 

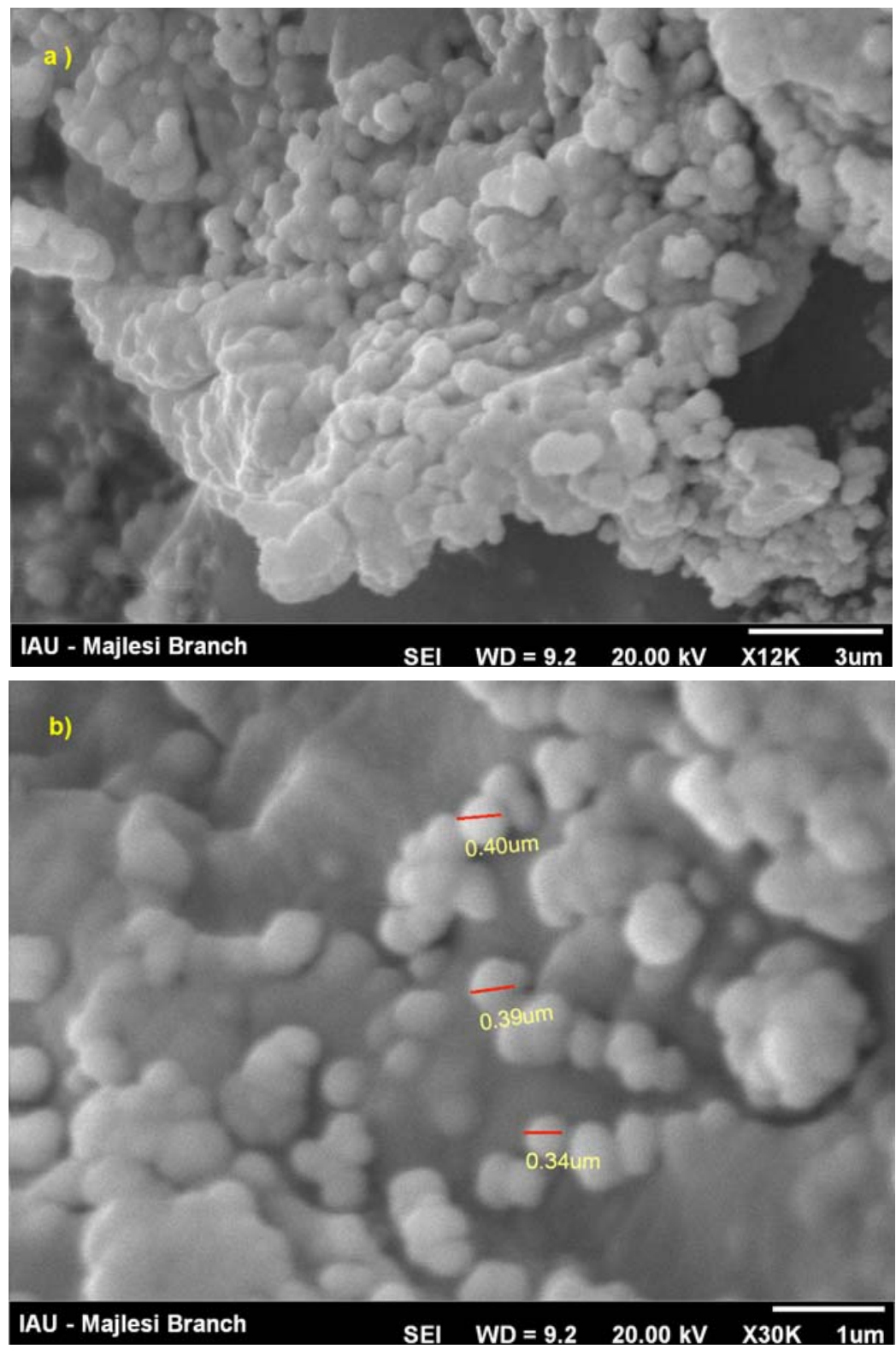

Figs. (7). a and b: SEM images of nano-ibuprofen with magnification of a) $12000 \mathrm{X}$ and b) $30000 \mathrm{X}$.

Table 1. Assay Calculation Results by 0.1 M Sodium Hydroxide Titration in Present of Phenolphthalein

\begin{tabular}{|c|c|c|c|}
\hline \multicolumn{2}{|c|}{ 0.1 M sodium hydroxide (ml) } & Nano-ibuprofen quantity in & Nano-ibuprofen purity (\%) \\
\hline \hline Blank & Nano-ibuprofen & 447.671 & 99.5 \\
\hline 0.1 & 21.8 & 445.608 & 99 \\
\hline 0.1 & 21.7 & 445.608 & 99 \\
\hline 0.1 & 21.7 & 449.734 & 99.9 \\
\hline 0.1 & 21.9 & 443.545 & 98.6 \\
\hline 0.1 & 21.6 & 446.4 & 99.2 \\
\hline & Mean & 2.35 & 0.52 \\
\hline
\end{tabular}




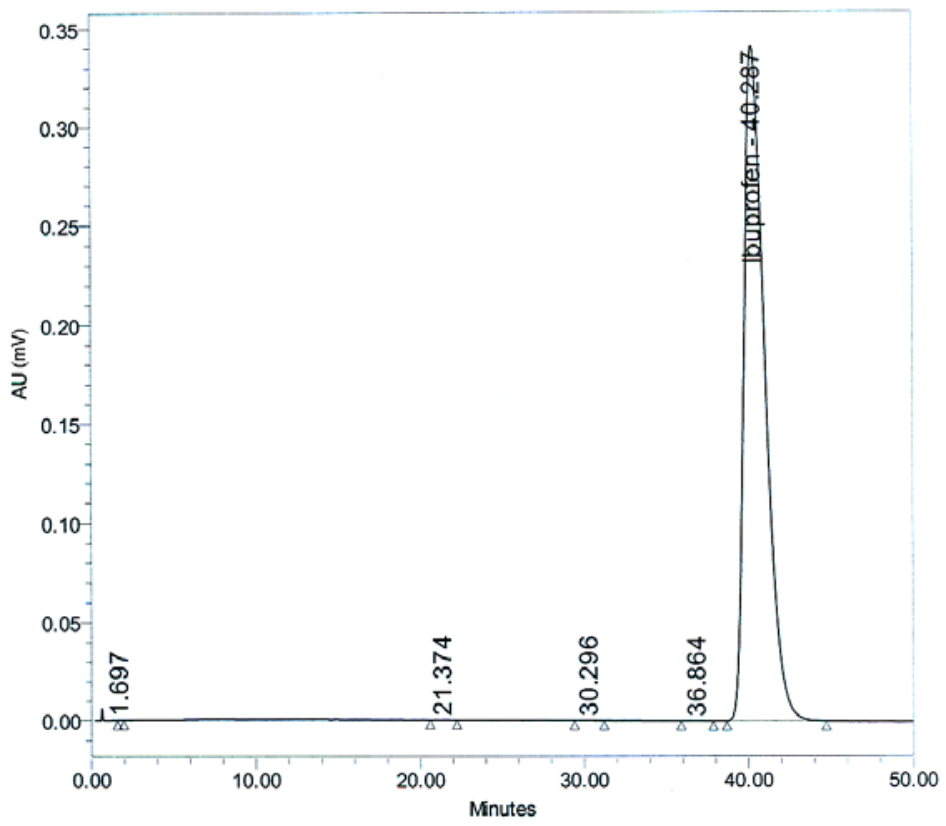

Fig. (8). HPLC chromatographic impurity spectrum for nano-ibuprofen.

Table 2. Nano-Ibuprofen Chromatographic Impurity Results

\begin{tabular}{|c|c|c|c|c|}
\hline Peak name & $\mathbf{r}_{\mathbf{i}}$ & $\mathbf{r}_{\mathbf{t}}$ & Individual impurity* & Total impurity** \\
\hline \hline & 1477 & 29996431 & 0.005 & \\
\hline & 5408 & & 0.018 & \\
\hline & 2588 & & 0.009 & 0.022 \\
\hline Ibuprofen & 29980264 & & & 0.1 \\
\hline
\end{tabular}

*Individual Impurity calculated by $100\left(\mathrm{r}_{\mathrm{i}} / \mathrm{r}_{\mathrm{t}}\right)$ other than the main ibuprofen peak

** Total impurity: the sum of the responses of all peaks other than the main ibuprofen peak.

before and after the precipitation process is the same and it is about +1.34 at $589.0 \mathrm{~nm}$.

\subsection{Dissolution Test}

Dissolution profile of raw ibuprofen and precipitated nanoparticles at room temperature are illustrated in Fig. (9), respectively. The dissolution rate of ibuprofen nanoparticles is 2.33 times that of raw drugs. According to NoyesWhitney equation, the solid dissolution rate is directly proportional to its surface area exposed to the dissolution medium. The accelerated dissolution for ibuprofen nanoparticles could thus be mainly ascribed to their greater surface area $\left(5.6-9.3 \mathrm{~m}^{2} / \mathrm{g}\right.$ ) in comparison with raw drug $\left(0.11-0.45 \mathrm{~m}^{2} / \mathrm{g}\right)$. This indicates that the acceleration of drug dissolution for nanoparticles occurs mostly in the first 30 min, after that, the dissolution has no significant difference from raw drugs.

\section{CONCLUSION}

Solvent/ antisolvent precipitation technique was employed to produce nanoparticles of ibuprofen, a poorly water-soluble drug, for the enhancement of solubility and dissolution rate. For this technique, the high supersaturated drug solution was prepared in isopropyl alcohol and purified

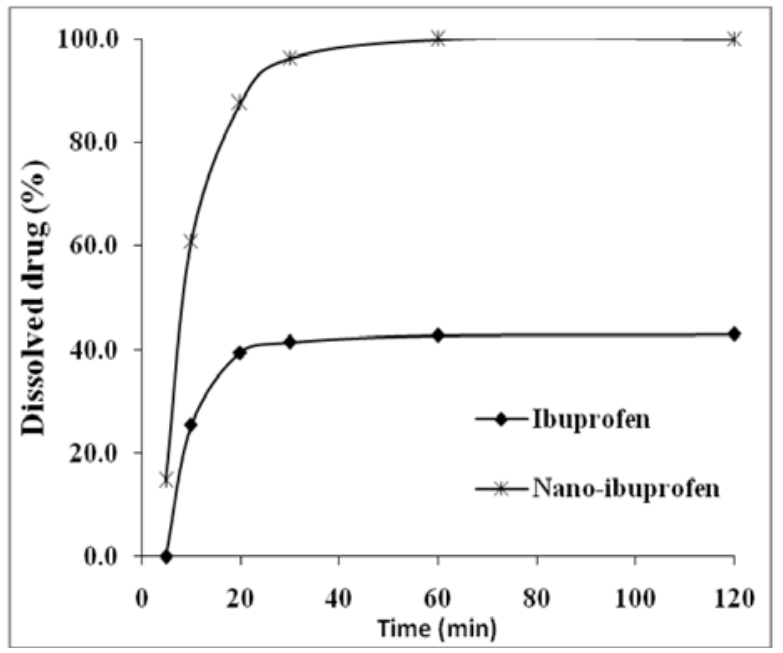

Fig. (9). Dissolution profile of raw ibuprofen and drug nanoibuprofen in purified water as media. 
water is used as the antisolvent. The sodium dodecyl sulfate and tri-ethanolamine as two stabilizers are used to prepare nanoparticles with size of 200- $400 \mathrm{~nm}$. The purity of prepared nanoparticles was $99.2 \pm 0.5 \%$. The acceleration of drug dissolution for nanoparticles occurs mostly in the first $30 \mathrm{~min}$ and is 2.33 times of raw drug. The chemical structure of ibuprofen is not show any change with decreasing of particles size. The specific optical rotation of nano-ibuprofen shows that the optical rotation of drug before and after the precipitation method is the same and it is about +1.34 at $589.0 \mathrm{~nm}$ sodium light. Antisolvent precipitation can thus be a simple and effective approach to produce nanoparticles of poor water soluble drugs.

\section{REFERENCES}

[1] Chowdhury, B.; Roy, D.; Chavan, U.; Mukhopadhyay, S. The antiinflammatory, antipyretic, analgesic compound ibuprofen also has antibacterial activity against gram-positive bacteria. Med. Sci. Res., 1996, 24, 801- 802 .

[2] Merisko, E.; Liversidge, G.G.; Cooper, E.R. Nanosizing: formulation approach for poorly-water-soluble compounds. Eur. J. Pharm. Sci., 2003, 18(2), 113-120.

[3] Lipinski, C.A. Poor aqueous solubility an industry wide problem in drug discovery. Am. Pharm. Rev., 2002, 53, 82.

[4] Ghosh, L.K.; Ghosh, N.C.; Chatterjee, M.; Gupta, B.K. Product development studies on the tablet formulation of ibuprofen to improve bioavailability. Drug Dev. Ind. Pharm., 1998, 24, 473477

[5] Vasconcelos, T.; Sarmento, B.; Costa, P. Solubility enhancement as strategies to improve oral bioavailability of poorly water soluble drugs. Drug Discovery Today, 2007, 12, 1281-1302.

[6] Lobenberg, R.; Amidon, G.L. Modern bioavailability and bioequivalence and biopharmaceutics classification system. New scientific approaches to international regulatory standards. Eur J. Pharm. Biopharm., 2000, 50, 3-12.

[7] Mosharraf, M.; Nystorm, C.; The effect of particle size and shape on the specific dissolution rate of micronized practically insoluble drugs. Int. J. Pharm., 1995, 122, 35.

[8] Liversidge, G.G.; Cundy, K.C. Particle size reduction for improvement of oral bioavailability of hydrophobic drugs: Absolute oral bioavailability of nanocrystalline Danzol in beagle dogs. Int. J. Pharm., 1995, 125, 91.

[9] Scholl, J.; Lindenberg, C.; Vicum, L.; Mazzotti, M. Antisolvent Precipitation of PDI 747: Kinetics of Particle Formation and Growth. J. Crys. Grow. Desi., 2007, 7(9), 1653-1661.

[10] Rajesh N.D.; Sameer, V.D. Controlling Particle Size of a Poorly Water-Soluble Drug Using Ultrasound and Stabilizers in Antisolvent Precipitation. Ind. Eng. Chem. Res., 2009, 48, 75817593.

[11] Sang-Do, Y.; Su-Jin, P.; Se-Yeoun, J. Recrystallization of a pharmaceutical compound using liquid and supercritical antisolvent. Ind. Eng. Chem. Res., 2006, 45, 2287-2293.

[12] Rasenack, N.; Steckel, H.; Muller, B.W. Preparation of microcrystals by in situ micronization. Powder Technol., 2004, 143, 291-296.
[13] Highton, F. In: The pharmaceutics of ibuprofen; Rainsford, K.D., Ed.; ibuprofen. A Critical Bibliographic Review, Taylor \& Francis: London, 1999, p. 53

[14] Newa, M.; Bhandari, K.H.; Kim, J.O.; Im, J.S.; Kim, J.A.; Yoo, B.K.; Woo, J.S.; Choi, H.G.; Yong, C.S. Enhancement of solubility, dissolution and bioavailability of ibuprofen in solid dispersion systems, Chem. Pharm. Bull., 2008, 56, 569-574.

[15] Noyes, A.A.; Whitney, W.R. The rate of solution of solid substances in their own solutions. J. Am. Chem. Soc., 1897, 19(12), 930-934

[16] Amidon, G.L.; Lennernas, H.; Shah, V.P.; Crison, J.R. A theoretical basis for a biopharmaceutical drug classification: The correlation of in vitro drug product dissolution and in vivo bioavailability. Pharm. Res., 1995, 12, 413.

[17] Liversidge, G.G.; Cundy, K.C. Particle size reduction for improvement of oral bioavailability of hydrophobic drugs: I. absolute oral bioavailability of nanocrystalline danazol in beagle dogs. Int. J. Pharm, 1995, 125, 91-97.

[18] Ghorab, M.K.; Adeyeye, M.C. Enhancement of ibuprofen dissolution via wet granulation with beta-cyclodextrin. Pharm. Dev. Technol., 2001, 6, 305-314.

[19] BP-2010, In: Ibuprofen; The department of health. British Pharmacopoeia Commission Office, Market Towers, The stationery office, London, 2009, Vol. 1, pp. 1089-1091.

[20] USP-32 $2^{\text {th }}$ edition, NF-2 $7^{\text {nd }}$ edition, In: ibuprofen; United state pharmacopeial convention. 12601, Twinbrook Parkway, Rockville, MD 20852, United book press, Maryland, 2008, Vol. 3, pp. 26072608.

[21] Matteucci, M.E.; Hotze, M.A.; Johnston, K.P.; Williams, R.O. Drug nanoparticles by antisolvent precipitation: mixing energy versus surfactant stabilization. Langmuir, 2006, 22, 8951-8959.

[22] Jian-Feng, C.; Ji-Yao, Z.; Zhi-Gang, S.; Jie, Z.; Jimmy, Y. Preparation and Characterization of Amorphous Cefuroxime Axetil Drug Nanoparticles with Novel Technology: High-Gravity Antisolvent Precipitation. Ind. Eng. Chem. Res., 2006, 45, 87238727.

[23] Yun, J.; Zhao, H.; Wang, J.; Wang, Q.; Chen, J. Controlled Liquid Antisolvent Precipitation of Hydrophobic Pharmaceutical Nanoparticles in a Microchannel Reactor. Ind. Eng. Chem. Res., 2007, 46, 8229-8235.

[24] Bergstrom, C.A.; Strafford, M.; Lazorava, L.; Avdeef, A.; Luthman, K.; Artursson, P. Absorption classification of oral drugs based on molecular surface properties. J. Med. Chem., 2003, 46, 558-570.

[25] Craig, D.Q.M. The mechanisms of drug release from solid dispersions in water-soluble polymers. Int. J. Pharm., 2002, 231, 131-144.

[26] Amidon, G.L.; Lennernas, H.; Shah, V.P.; Crison, J.R. A theoretical basis for a biopharmaceutical drug classification: The correlation of in-vitro drug product dissolution and in vivo bioavailability. Pharm. Res., 1995, 12, 413-419.

[27] Levy, G. Effect of particle size on dissolution and gastrointestinal absorption rates of pharmaceuticals. Am. J. Pharm. Sci., 1963, 135, 78-92.

[28] Serajuddin, A.T.M. Solid Dispersion of Poorly Water Soluble Drugs: Early Promises, Subsequent Problems, and Recent Breakthrough. J. Pharm. Sci., 1999, 88, 1058-1066.

(C) Mansouri et al.; Licensee Bentham Open.

This is an open access article licensed under the terms of the Creative Commons Attribution Non-Commercial License (http://creativecommons.org/licenses/by-nc/3.0/) which permits unrestricted, non-commercial use, distribution and reproduction in any medium, provided the work is properly cited. 